\title{
Guidelines for the Investigation and Management of Ground Glass Nodules
}

\author{
Jun Hee Lee, M.D., Jeong In Hong, M.D., Hyun Koo Kim, M.D., Ph.D. \\ Department of Thoracic and Cardiovascular Surgery, Korea University Guro Hospital, Korea University College of Medicine, Seoul, Korea
}

\section{ARTICLE INFO}

Received March 8, 2021

Revised April 30, 2021

Accepted June 3, 2021

\section{Corresponding author}

Hyun Koo Kim

Tel 82-2-2626-3106

Fax 82-2-866-6377

E-mail kimhyunkoo@korea.ac.kr

ORCID

https://orcid.org/0000-0001-7604-4729

TThis article was presented at the 52nd Autumn Annual Conference of the Thoracic and Cardiovascular Surgery, Seoul, Korea, on November 5-7, 2020.

\begin{abstract}
The clinical significance of ground-glass nodules (GGNs) has been investigated in extensive clinical research for many years. The natural history of GGNs is known to be closely related to their size, proportion of solid components, and size progression over time. Based on these data, several guidelines for GGN management have been published worldwide. The indications for nonsurgical biopsy or surgical resection of GGNs are as follows: pure GGNs between 5 and $10 \mathrm{~mm}$ in size if they increase in size or show development of a solid component at follow-up, pure GGNs $>10-15 \mathrm{~mm}$ that remain stable but persistent, part-solid nodules $>8 \mathrm{~mm}$ persisting at follow-up, or part-solid nodules with a solid component $>6 \mathrm{~mm}$ at follow-up. Newly updated data considering geographical or racial factors and recent developments in surgical techniques may improve the surgical indications for GGNs in the near future.
\end{abstract}

Keywords: Ground glass nodules, Preinvasive lung cancer, Non-small cell lung cancer, Guideline, Surgery

\section{Introduction}

The importance of the early diagnosis of lung cancer was proven in the National Lung Screening Trial, which showed that low-dose computed tomography (CT) in highrisk individuals reduced the mortality rate of lung cancer [1]. Based on this trial, the US Preventive Services Task Forces and the Ministry of Health and Welfare of South Korea updated their guidelines for lung cancer screening to expand the indications for early diagnosis [2]. This increased utilization of CT has led to the discovery of large numbers of ground-glass nodules (GGNs) and solitary nodules [3].

The clinical significance of GGNs has been investigated in extensive clinical research for many years. The natural history of GGNs is known to be closely related to their size, proportion of solid components, and size progression over time [3-5]. Based on these data, several guidelines for GGN management have been provided worldwide.

However, recent advancements in technology for detecting or managing GGNs with unsolved issues may lead to guideline updates. In particular, recent developments in surgical techniques have driven amendments to these surgery-oriented guidelines to offer patients the best treatment options [3].

\section{Japanese Society for CT Screening}

The Japanese Society for CT Screening guidelines were devised from a radiology/CT perspective [6]. All patients are subjected to CT surveillance. Biopsy or surgery is reserved for lesions that are $>15 \mathrm{~mm}$ in size and have grown since the follow-up visit or have developed a solid component $>5 \mathrm{~mm}$ in size. Intriguingly, these guidelines allow individual hospitals to proceed to biopsy or surgery for smaller part-solid nodules even if the solid component is $\leq 5 \mathrm{~mm}$, perhaps reflective of the greater concern over the probability of malignancy in GGNs in East Asia [7].

\section{American College of Chest Physicians guidelines}

The American College of Chest Physicians guidelines provide a slightly more multidisciplinary perspective $[3,8]$. 
Pure GGNs $\leq 5 \mathrm{~mm}$ in size require no further evaluation, while pure GGNs $>5 \mathrm{~mm}$ require annual surveillance with chest CT for at least 3 years.

Biopsy or surgery is performed for pure GGNs that grow or develop a solid component during follow-up. If they are pure and $>10 \mathrm{~mm}$ in size on initial CT, early follow-up at 3 months may be indicated for nonsolid nodules $>10 \mathrm{~mm}$ in size (followed by nonsurgical biopsy and/or surgical resection for persistent nodules).

A part-solid nodule $\leq 8 \mathrm{~mm}$ in size requires CT surveillance at approximately 3,12 , and 24 months, followed by annual CT surveillance for an additional 1-3 years. A part-solid nodule $>8 \mathrm{~mm}$ requires repeat chest $\mathrm{CT}$ at 3 months, followed by further evaluation with positron emission tomography (PET), nonsurgical biopsy, and/or surgical resection if persistent. Part-solid nodules $>15 \mathrm{~mm}$ on presentation should directly proceed to further evaluation with PET-CT, nonsurgical biopsy, and/or surgical resection.

\section{British Thoracic Society guidelines}

The British Thoracic Society guidelines provide more clarity on the use of further imaging, with ordinal scale reporting for PET-CT recommended to facilitate incorporation into risk models and more clarity on the location of the biopsy. It contains recommendations for the threshold for treatment without histological confirmation [9].

GGNs $<5 \mathrm{~mm}$ in size that are known to have been stable for over 4 years do not require follow-up, whereas all others require repeat CT at 3 months. GGNs that persist and have morphological features that are suggestive of malignancy can be subjected to surgical excision. For GGNs that show growth or an altered morphology, surgical resection is mandatory.

\section{Asian consensus guidelines}

In 2016, the Asian consensus guidelines were developed because of the increasing incidence and distinctive characteristics observed in patients with lung cancer in Asia [10]. For the evaluation of pulmonary nodules in Asian patients, clinicians may consider data unique to Asian patients, such as exposure to air pollution, a higher smoking rate, and a high prevalence of granulomatous disease and other infectious causes of pulmonary nodules. The key differences between the Asian consensus guidelines and other guidelines are the considerations for the extended surveillance of nodules and the greater emphasis on nonsurgical biopsy instead of PET-CT due to the high prevalence of tuberculosis.

Solitary, smaller pure GGNs $(<5 \mathrm{~mm})$ require no follow-up surveillance CT, whereas solitary, larger pure GGNs $(\geq 5 \mathrm{~mm}$ ) require annual follow-up surveillance CT for at least 3 years. Clinicians should consider ongoing annual surveillance CT depending on a discussion with the patient.

Solitary, smaller part-solid nodules $(<8 \mathrm{~mm})$ require follow-up CT examinations at 3,12, and 24 months and then annually thereafter. Solitary, larger part-solid nodules $(\geq 8$ $\mathrm{mm}$ ) require initial follow-up surveillance CT at 3 months, with consideration for antimicrobial therapy. If nodules persist beyond 3 months, nonsurgical biopsy and/or surgical biopsy is recommended, and PET-CT is an additional option for the staging of the disease before surgical resection. Clinicians should consider ongoing annual surveillance CT depending on a discussion with the patient.

\section{Fleischner Society guidelines}

The Fleischner Society first published recommendations for the management of small pulmonary nodules in a statement in 2005 [11]; these recommendations were subsequently updated in 2017 to overcome important limitations, particularly the insufficiently detailed consideration of subsolid lung nodules [12].

Lesions should be established as true GGNs, preferably with the use of contiguous thin CT sections ( $1 \mathrm{~mm}$ thick). Solitary, smaller pure GGNs ( $<6 \mathrm{~mm}$ ) require no follow-up surveillance CT. Solitary, larger pure GGNs $(\geq 6 \mathrm{~mm})$ require initial follow-up surveillance CT within 6-12 months to determine persistence, followed by surveillance CT every 2 years until 5 years if they remain persistent and unchanged.

Solitary, smaller part-solid nodules $(<6 \mathrm{~mm})$ require no follow-up surveillance CT, whereas solitary, larger part-solid nodules ( $\geq 6 \mathrm{~mm}$ ) require initial follow-up CT within 3-6 months to determine persistence. If they remain persistent and the solid component is $<6 \mathrm{~mm}$ in size, annual surveillance CT is performed for a minimum of 5 years. PET/CT, nonsurgical biopsy, or surgical resection is recommended for nodules with suspicious features (lobulated margins or cystic components), a developing solid component, or a large solid component ( $\geq 8 \mathrm{~mm}$ ).

Multiple smaller subsolid nodules $(<6 \mathrm{~mm})$ require initial follow-up surveillance CT within 3-6 months, followed by surveillance CT at 2 and 4 years if they remain persistent and unchanged. Multiple larger subsolid nodules $(\geq 6$ 


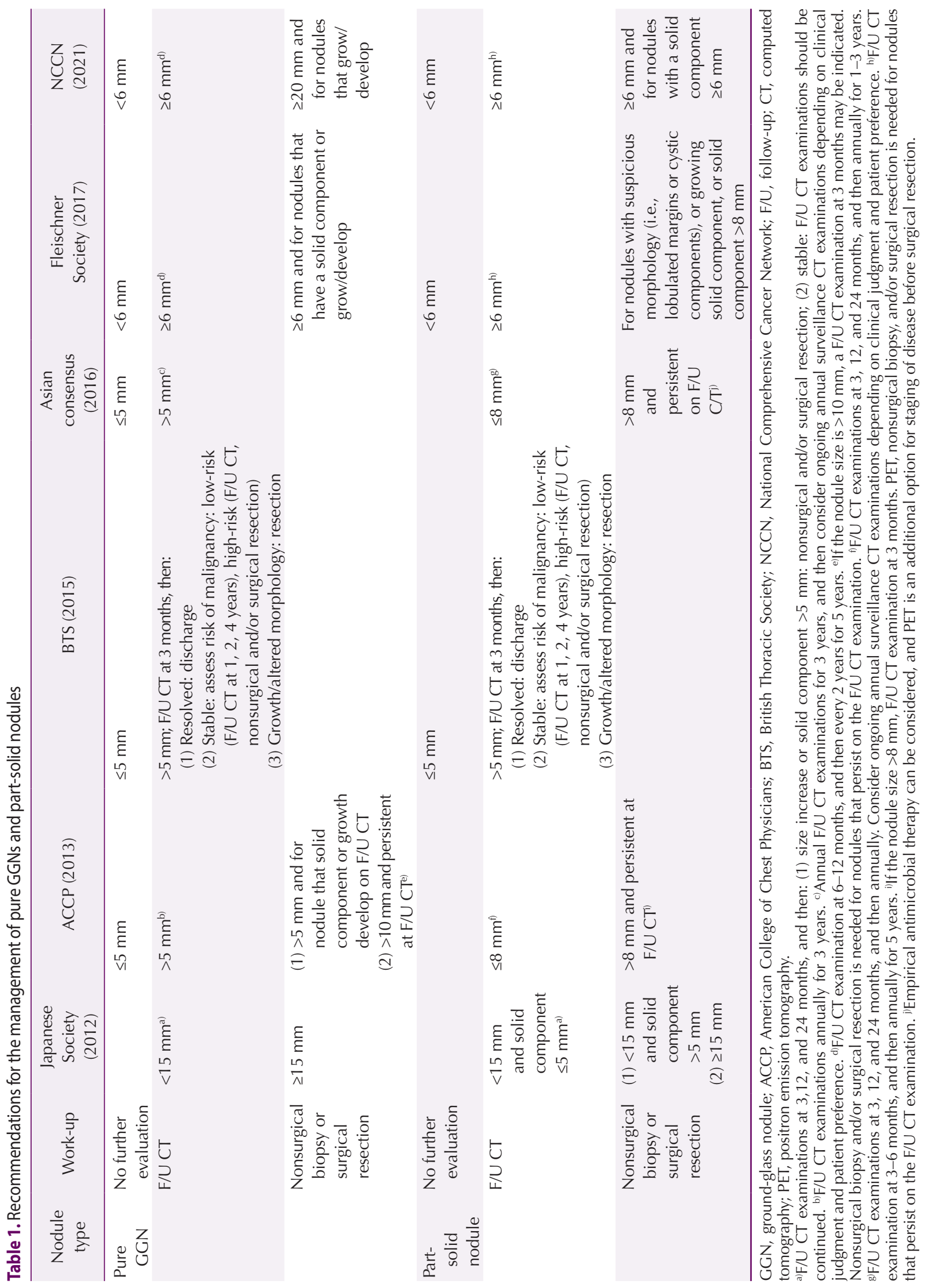


$\mathrm{mm}$ ) also require initial follow-up surveillance CT within 3-6 months, and the most suspicious nodule will determine subsequent management.

\section{National Comprehensive Cancer Network guidelines}

The National Comprehensive Cancer Network guidelines, which are drafted by an intentionally multidisciplinary panel, are possibly the most widely used and referenced [13]. Solitary smaller pure GGNs and part-sloid nodules $(<6 \mathrm{~mm})$ require no follow-up surveillance $\mathrm{CT}$, whereas solitary larger pure GGNs ( $\geq 6 \mathrm{~mm}$ ) require an initial follow-up surveillance CT within 6-12 months, followed by surveillance CT every 2 years until 5 years. Solitary larger part-solid nodules $(\geq 6 \mathrm{~mm})$ require follow-up surveillance CT within 3-6 months. PET-CT, biopsy, or surgical resection is considered for nodules with a solid component $\geq 6 \mathrm{~mm}$ in size.

\section{Lung Imaging Reporting and Data System}

The Lung Imaging Reporting and Data System (LungRADS) is not a guideline, but a standardized reporting tool. In 2014, the American College of Radiology developed Lung-RADS ver. 1.0 (American College of Radiology, Reston, VA, USA) to standardize lung cancer screening CT reporting, and management recommendations [14]. LungRADS ver. 1.0 (American College of Radiology) was modified and adopted for the national lung cancer screening program in Korea. After the system was revised, LungRADS ver. 1.1 (American College of Radiology) was published in 2019 [15].

The Lung-RADS scoring system is classified into categories 1-4 according to the nodule consistency (solid, part-solid, and ground-glass), size, and growth (increase in nodule size $>1.5 \mathrm{~mm}$ ). Lung-RADS category 4 is further divided into 3 subcategories (4-A, 4-B, and 4-X) according to the nature of the nodule. As a modifier, the letter " $\mathrm{S}$ " is added to categories 0-4 coding (e.g., 4AS, 4BS) to describe any clinically significant incidental findings.

Pure GGNs $(\geq 30 \mathrm{~mm})$ are classified into category 3 (probably benign) and part-solid nodules ( $\geq 6 \mathrm{~mm}$ ) with a solid component $\geq 6 \mathrm{~mm}$ in size are classified into category 4 (suspicious).

\section{Conclusion}

The guidelines for GGNs are inconsistent and still require updating as data continue to be gathered on the natural history of GGNs. The current guidelines for pure GGNs and part-solid nodules are summarized in Table 1.

Based on the present information provided, the indications for nonsurgical biopsy or the surgical resection of GGNs are as follows: pure GGNs between 5 and $10 \mathrm{~mm}$ in size if they increase in size or develop a solid component at follow-up, pure GGNs $>10-15 \mathrm{~mm}$ that remain stable but persistent, part-solid nodules $>8 \mathrm{~mm}$ persisting at follow-up, or part-solid nodules with a solid component $>6$ $\mathrm{mm}$ at follow-up. Newly updated data considering geographical or racial factors and recent developments in surgical techniques may improve the surgical indications for GGNs in the near future.

\section{Conflict of interest}

No potential conflict of interest relevant to this article was reported.

\section{Funding}

This study was supported by the Korean Health Technology R\&D Project, Ministry of Health and Welfare, Republic of Korea (ID: HI19C0166).

\section{ORCID}

Jun Hee Lee: https://orcid.org/0000-0002-6592-6483

Jeong In Hong: https://orcid.org/0000-0002-9660-7063

Hyun Koo Kim: https://orcid.org/0000-0001-7604-4729

\section{References}

1. National Lung Screening Trial Research Team, Church TR, Black WC, et al. Results of initial low-dose computed tomographic screening for lung cancer. N Engl J Med 2013;368:1980-91.

2. Kim Y, Rho J, Quan YH, et al. Simultaneous visualization of pulmonary nodules and intersegmental planes on fluorescent images in pulmonary segmentectomy. Eur J Cardiothorac Surg 2020;58(Suppl_1):i77-84.

3. Sihoe ADL, Cardillo G. Solitary pulmonary ground-glass opacity: is it time for new surgical guidelines? Eur J Cardiothorac Surg 2017; 52:848-51.

4. Chang B, Hwang JH, Choi YH, et al. Natural history of pure groundglass opacity lung nodules detected by low-dose CT scan. Chest 


\section{$2013 ; 143: 172-8$}

5. Takashima S, Maruyama Y, Hasegawa M, et al. CT findings and progression of small peripheral lung neoplasms having a replacement growth pattern. AJR Am J Roentgenol 2003;180:817-26.

6. Japanese Society for CT Screening. Guidelines for the management of pulmonary nodules detected by low-dose CT lung cancer screening version 3 [Internet]. Tokyo: Japanese Society for CT Screening; 2013 [cited 2017 Feb 3]. Available from: http://www.jscts.org/pdf/ guideline/gls3rd_english130621.pdf.

7. Nawa T, Nakagawa T, Mizoue T, Endo K. Low-dose computed tomography screening in Japan. J Thorac Imaging 2015;30:108-14.

8. Gould MK, Donington J, Lynch WR, et al. Evaluation of individuals with pulmonary nodules: when is it lung cancer?: diagnosis and management of lung cancer, 3rd ed: American College of Chest Physicians evidence-based clinical practice guidelines. Chest 2013;143(5 Suppl):e93S-e120S

9. Callister ME, Baldwin DR, Akram AR, et al. British Thoracic Society guidelines for the investigation and management of pulmonary nodules. Thorax 2015;70 Suppl 2:ii1-54.

10. Bai C, Choi CM, Chu CM, et al. Evaluation of pulmonary nodules: clinical practice consensus guidelines for Asia. Chest 2016;150:877-
93.

11. MacMahon H, Austin JH, Gamsu G, et al. Guidelines for management of small pulmonary nodules detected on CT scans: a statement from the Fleischner Society. Radiology 2005;237:395-400.

12. MacMahon H, Naidich DP, Goo JM, et al. Guidelines for management of incidental pulmonary nodules detected on CT images: from the Fleischner Society 2017. Radiology 2017;284:228-43.

13. National Comprehensive Cancer Network. NCCN clinical practice guidelines in oncology: lung cancer screening version 4.2021 [Internet]. Plymouth Meeting (PA): National Comprehensive Cancer Network; 2021 [cited 2021 Mar 3]. Available from: https://www.ncen. org/guidelines/recently-published-guidelines.

14. American College of Radiology. Lung CT screening reporting \& data system (Lung-RADS) [Internet]. Reston (VA): American College of Radiology; c2014 [cited 2019 May 12]. Available from: https://www. acr.org/Clinical-Resources/Reportingand-Data-systems/Lung-Rads.

15. American College of Radiology. Lung CT screening reporting \& data system 2019: Lung-RADS version 1.1 [Internet]. Reston (VA): American College of Radiology; 2019 [cited 2019 Jul 6]. Available from: https://www.acr.org/-/media/ACR/Files/RADS/Lung-RADS/ LungRADSAssessmentCategoriesv1-1.pdf?la=en. 